\title{
La disputa sobre el aborto en México: Discursos contrastados de personificación, derechos, la familia y el Estado*
}

\section{The dispute over abortion in Mexico: Contrasted speeches of personification, rights, family and the State}

\section{A disputa sobre aborto no México: Discursos contrastados de personificação, direitos, família e Estado*}

Fecha de recepción: 24-08-15 Fecha de aceptación: 10-09-15 Disponible en línea: 01-11-15 doi: 10.11144/Javeriana.rgyps14-29.dsam

Cómo citar este artículo:

Maier E. La disputa sobre el aborto en México: Discursos contrastados de personificación, derechos, la familia y el Estado. Rev. Gerenc. Polít. Salud. 2015; 14(29): 10-24. http://dx.doi.org/10.11144/Javeriana. rgyps14-29.dsam 


\section{Resumen}

El presente artículo explora las posturas enfrentadas sobre el aborto en México, mediante el análisis de contenido de textos y pronunciamientos publicados recientemente y emitidos por actores colectivos nacionales y sus aliados o institutos internacionales. A partir del estudio de las diferencias de significación del aborto en los discursos en pugna en México hoy en día, no solo se pretende identificar las lógicas discursivas diferenciales que los sustentan, sino también situar esta disputa en el contexto de la era actual de transición entre dos modelos estructuralestecnológicos capitalistas: la modernidad industrial y el postindustrialismo, y de tal manera argumentar que el aborto es emblemático de una contienda contemporánea mayor por la propia naturaleza del Estado y su relación con la religión. En este sentido, el enfrentamiento sobre el aborto es también una disputa por el proyecto societario.

Palabras clave: aborto; discursos; México; disputa; guerra cultural; proyecto societario

\section{Abstract}

This article explores the opposing positions on abortion in Mexico, using the content analysis of texts and pronouncements recently published and issued by domestic collective actors and their allies or international institutes. From the study of the differences of significance of abortion in rivaled speeches in Mexico today, not only does this article seek to identify the distinguishing discursive logic that supports them, but also put this dispute in the context of the current era of transition between two capitalist structural-technological models: industrial modernity and post-industrialism, and so argue that abortion is emblematic of a larger contemporary race for the nature of the state and its relationship with religion. As such, it represents a clash over the societal project itself.

Keywords: abortion; discourses; Mexico; dispute; culture war; societal project

\section{Resumo}

O presente artigo explora as posturas enfrentadas sobre o aborto no México, mediante análise de conteúdo de textos e pronunciamentos publicados recentemente e emitidos por atores coletivos nacionais e seus aliados ou institutos internacionais. A partir do estudo das diferenças de significação do aborto nos discursos concorrentes no México de hoje, não apenas objetiva-se identificar as lógicas discursivas diferenciais que os sustentam, mas também colocar tal disputa no contexto da era atual de transição entre dois modelos estruturais-tecnológicos capitalistas: a modernidade industrial e o pós-industrialismo, e de tal forma argumentar que o aborto é emblemático de uma contenda contemporânea maior pela própria natureza do Estado e seu relacionamento com a religião. Neste sentido, o confronto sobre aborto é também uma disputa pelo projeto societário.

Palavras-chave: aborto; discursos; México; disputa; guerra cultural; projeto societário 


\section{Introducción}

El aborto es un tema espinoso. Su simple mención suele generar confrontaciones apasionadas entre posiciones ancladas a universos simbólicos radicalmente distintos y hasta contrapuestos. Cada universo simbólico se sustenta en una hermenéutica que entiende los discursos en pugna, traza la evolución de los actores colectivos contrarios e interpreta sus acciones desde miradas paradigmáticas profundamente disímiles y también competidas. En este sentido, las distintas lecturas del aborto develan una complejidad temática que no solo evoca emociones intensas, sino que además pone a estas interpretaciones en un estado de urgencia, al fusionar los discursos, actores y acciones enfrentadas en una dialéctica constructora de verdades, de vida y muerte. Asimismo, las premisas discursivas que construyen los respectivos sentidos del aborto encierran tácticas y estrategias de poder, que desde miradas ontológicas discordantes, disputan los significados de la vida, la persona, la familia, las identidades y arreglos de género y la relación entre religión y gobernanza, constituyéndose así en archipiélagos de saberes y verdades excluyentes e intensamente asumidos, interiorizados y defendidos por sus defensores (1).

En México dicha contienda se recrudeció en el 2007 a partir de la despenalización del aborto en el D.F. durante el primer trimestre de embarazo. En reacción a la reforma de política sanitaria en la ciudad capital, se orquestó a nivel nacional una estrategia conservadora para blindarse contra intentos similares de despenalización en el resto de los estados de la República, mediante la legislación de reformas constitucionales locales que avalan el derecho a la vida desde la concepción. Estas últimas reformas se traducen legalmente en la personificación del cigoto desde la fase precelular, lo que resulta en la tipificación implícita de asesina- to premeditado a la interrupción intencional del embarazo en cualquier momento de la gestación y la criminalización de las mujeres que aborten. ${ }^{1}$

Ambas partes en disputa defienden sus acciones y políticas a partir de su propia lógica discursiva, sustentada en sus respectivas fuentes de autoridad y premisas morales. En el caso del discurso de los derechos reproductivos, se basa en una lógica laica, científico-sanitaria, de autonomía corporal, libertad de conciencia, decisión y de justicia distributiva. Por el otro lado, el discurso conservador-tradicionalista descansa en una lógica religiosa, jerárquica, de derechos divinos y verdades absolutas, naturalizadas, predeterminadas y presociales.

El propósito del presente artículo es examinar las posturas mencionadas de la disputa sobre el aborto en México, mediante el análisis de contenido de informes y pronunciamientos publicados recientemente y emitidos por actores colectivos nacionales y sus aliados o institutos internacionales. El texto representa un avance del trabajo de investigación realizado por la autora en los últimos años, dedicado a analizar las diferencias interpretativas del aborto tanto en México como en Estados Unidos, ${ }^{2}$ identificar sus lógicas internas y explorar sus implicaciones en el contexto de transición entre modelos estructurales y tecnológicos que define nuestra era contemporánea. ${ }^{3}$ En este sentido, se contrastan analíticamente las diferencias discursivas atribuidas a la termi-

1 En la mayoría de los estados que legislaron dichas reformas existen excepciones por violación y amenaza a la vida de la madre, en otros, por incesto; solo Baja California no considera excepción alguna.

2 El análisis presentado en el presente artículo formará parte del capítulo sobre México del libro que la autora actualmente escribe sobre dicho tema en ambos países, cuya publicación se proyecta para el 2017.

3 Véase Elizabeth Maier (2-6). 
nación voluntaria del embarazo en México en los informes y pronunciamientos analizados, lo que finalmente devela la complejidad de lo que está en juego en el debate.

A partir de la tesis expuesta al inicio del siguiente apartado, se caracteriza el contexto estructural contemporáneo, por considerárselo imprescindible para comprender la amplitud de las implicaciones de la disputa en pro o en contra de la interrupción intencional del embarazo. En el tercer apartado se analizan textos y pronunciamientos emitidos por los actores colectivos representativos de los discursos en contención, destacando sus premisas, valores y visión del mundo, con énfasis en la dialéctica entre contexto, actores y discursos. Finalmente, en el apartado de conclusión, se reflexiona sobre las implicaciones de la controversia que propicia el aborto en México, con interrogantes en torno a la tensión entre el Estado-nación secular de la modernidad y lo que apunta ahora hacia un Estado postsecular con creciente influencia de la religión.

\section{Contextualizando la disputa discursiva sobre el aborto en México}

La tesis que orienta el presente análisis comprende la disputa por el sentido del aborto ${ }^{4}$ como emblemática de una contienda histórica mayor, producto del contexto contemporáneo de transición entre dos modelos del capitalismo, es decir, entre la modernidad industrial del siglo pasado y la actual era neoliberal postindustrial globalizada. Las implicaciones de los marcados avances tecnológicos en materia de las comunicaciones, la robótica, así como su implícita resignifica-

4 Asimismo, dicha contienda circunscribe el cuerpo de la mujer, la sexualidad y la reproducción como campos también en disputa. ción de la noción espacio-tiempo, ${ }^{5}$ la reorganización productiva, el cambio de las reglas y espacios de trabajo, la separación relativa del mundo de la producción de las finanzas, la reconfiguración de las relaciones sociales - como la de género-, la reestructuración de la pirámide de las clases sociales y, sobre todo, la creciente pérdida de cohesión y eficacia de las instituciones sociales de la era industrial, se traducen a un escenario general movedizo, de cambios constantes, fluctuación e incertidumbre, en donde la tensión entre el desfase de las instituciones rebasadas de la pasada era industrial y lo líquido de la vida cotidiana postindustrial (8), abre el campo de disputa por la redefinición institucional, simultáneamente por el privilegio de la interpretación cultural $(9,10)$.

El desencuentro entre los nuevos acomodos y prácticas de la vida cotidiana postindustrial y los anteriores arreglos y valores institucionales fragmenta la reciprocidad anterior entre lo productivo, lo político y lo cultural, que Beck (10) considera un rasgo distintivo de la reproducción de la modernidad industrial. Fue "un modelo de vida en el que los papeles de los sexos, la unidad familiar y las clases forman parte de la misma cadena" (10, p. 14), reproduciendo así el modelo industrial como sistema integral. El decaimiento de dicho modelo y su paulatino reemplazo por la sociedad liquida involucra a dos fenómenos interrelacionados fundamentales, ambos productos del contexto coyuntural de transición entre modelos capitalistas: la lucha feminista por la igualdad de género, por un lado, y la creciente incorporación de las mujeres al empleo formal, por el otro. Dicho proceso de sujetivización de las mujeres trajo consigo nuevas reclamaciones; central en

5 Castells (7) llama a dicha reconfiguración del tiempo y del espacio de la era de redes cibernéticas e información, el "tiempo sin tiempo y el espacio de los flujos", indicando que traen implícitos rearreglos de poder propios de la nueva época (p. 34). 
ellas fue el tema de la autonomía corporal, es decir, el derecho de decidir sobre su capacidad reproductiva y determinar cuántos hijos tener y cuándo tenerlos.

Situado en este contexto de tensión entre el desfasamiento institucional y la pugna contemporánea por los sentidos culturales, el aborto se vuelve un tema altamente contencioso por su importancia en la definición de una serie de factores fundamentales en la definición de nuevos contenidos institucionales y del propio rumbo del proyecto societal. Destacan entre los más relevantes: el momento de la personificación y de ser sujeto a derechos; la definición y sujeto de la ciudadanía; la interpretación de los derechos humanos; el perfil de la familia; y los rasgos del Estado en cuanto a su relación con la religión. En este sentido, la interrupción voluntaria del embarazo encierra conflictos de mucha mayor envergadura, emanados de los desajustes institucionales que hoy en día anuncian nuevas contiendas por el proyecto nacional mismo. De tal manera, en esta fase temprana de la era postindustrial se revisan y se redisputan algunos de los grandes fundamentos de la modernidad, pero a diferencia de la pasada época industrial, hoy el cuerpo reproductor de las mujeres y el cuerpo del deseo son los terrenos de batalla donde - como en el caso del aborto- discursos competidos proyectan significados distintos para un mismo referente, construyendo valores, códigos morales y legales, pautas de comportamiento, nociones de nación e imaginarios societales profundamente disimiles y enfrentados.

Hunter y Wolfe (11) se refieren a los vaivenes de este conflicto contemporáneo en los Estados Unidos como "la guerra cultural", haciendo alusión con ello al concepto alemán del siglo XVIII que se remitía a la contienda principal

14 de la temprana modernidad -0 modernidad simple, como la llama Beck (10)_, entre tradi- cionalistas religiosos y liberales laicos por el carácter del Estado y el modelo de organización social. En cuanto al conflicto actual, Hunter enfatiza la condición contextual y competida de lo cultural, señalando que "el poder de la cultura es el poder de nombrar las cosas, de definir la realidad y moldear los mundos de sentido. A su mayor alcance (la cultura) es el poder de proyectar la propia visión del mundo como la dominante, la única legítima, de manera que se vuelve incuestionable" (10, p. 15). En palabras de Bourdieu (que recupera Hussel), es lo “dóxico", es decir, la percepción de una verdad irrefutable que legitime el sistema de creencias profundas de los individuos (12). Y si la cultura siempre es un campo contestado entre miradas opuestas o binarias de lo que se considera real y deseable y los medios que permiten conocer lo real y deseable (11), finalmente, la disputa por la interpretación cultural es la contienda - entre élites, dice Hunter- por las posiciones, los recursos y el capital simbólico que otorgue el poder. A esto - refiriéndose a las clases sociales- Gramsci lo entendió como la lucha por la hegemonía (13).

Hunter (14) distingue entre cultura privada ${ }^{6}$ y cultura pública, sostiene que esta última es el espacio privilegiado del conflicto cultural por ser el locus de símbolos y significados que cohesionan la vida comunitaria, regional o nacional. De hecho, en los tiempos industriales modernos $-\mathrm{y}$, en parte, podríamos inferir que en los postindustriales también - la cultura pública se inserta en el contexto del Estado-Nación, los códigos, normas, leyes, símbolos, emblemas, mitos de

6 El autor entiende la cultura privada como el universo simbólico que articula la manera en que las personas se comprenden a sí mismas y se relacionan con los otros cercanos, cómo se organizan en su vida privada e íntima y qué recursos emplean para navegar los acontecimientos cotidianos. En fin, la cultura privada — según Hunter- es aquella que permite ubicar sus propias biografías y las biografías de las personas cercanas, en el contexto mayor de la comunidad, la nación, la historia humana, el planeta y el cosmos (11). 
origen y modos de vida colectiva que definan la identidad nacional, los significados de la ciudadanía, así como el imaginario colectivo de Nación. Generalmente, existe una interrelación dialéctica entre ambos orbes culturales, fortaleciéndose mutuamente y creando la sensación dóxica, a pesar de las siempre presentes tensiones entre visiones contrastadas del mundo. Pero en momentos históricos de transición y contextos cambiantes — como los actuales- la interpretación hegemónica resiente mayores fisuras y la irrupción de nuevos actores con nuevos discursos intensifica la contienda por la hegemonía de los significados culturales. Y en nuestro caso, transformando la cultura privada en sustancia de disputa pública, como bien lo apuntó el feminismo de la segunda ola con su lema "lo personal es político".

El discurso es el vehículo que articula, divulga y confronta las miradas societales contendientes, siendo uno de los dispositivos que garantizan el ejercicio del poder, en este caso mediante la construcción de significados que informan el pensar, actuar y sentir de los actores colectivos (15). ${ }^{7}$ Siempre inscrito en una formación histórico-discursiva y culturalmente específica que define su proceso de producción, la posición social de sus emisores y su jerarquización en cuanto a la hegemonía interpretativa, cada discurso es "un mensaje situado" que habla de la posición de los sujetos en la estructura social, así como de su coyuntura histórica, enraizándose en las relaciones de poder de una sociedad particular, adquiriendo sentido por su posicionamiento con relación a otros discursos (16).

En el actual contexto postindustrial de creciente interconectividad global, se hace énfasis en las posibilidades del discurso transnacionalizado, que en referencia al discurso

7 El otro dispositivo - según el autor — es la coerción (o su posibilidad). feminista, Álvarez (17) define como "el uso de marcos discursivos y prácticas organizacionales y políticas que son inspiradas y reafirmadas - aunque no necesariamente causadas - por la interrelación con actores sociales más allá de las fronteras nacionales, a través de un amplio rango de contactos, discusiones, transacciones y redes, tanto virtuales como materiales". ${ }^{8}$ La posibilidad que brindan las nuevas tecnologías de compactar el espacio-tiempo y hacer del globo entero un campo comunicativo continuo entre discursos, experiencias y estrategias de emisores colectivos, que en una multiplicidad de realidades locales concretas representan adaptaciones específicas de los discursos en contención. En este sentido, la contienda actual por los significados del aborto en México se sustenta en y a la vez informa pasajes ideológicos globales, que hoy en día disputan los grados de influencia de las religiones en la cultura pública de las naciones, lo que finalmente determina el perfil del Estado y su posicionamiento en esta misma contienda por la hegemonía de la voluntad colectiva mundial en las instituciones internacionales e interestatales.

\section{Discursos transnacionalizados de la vida, el aborto y los derechos}

\section{Introducción}

En México, la diputa por cómo interpretar el aborto se intensificó a finales de 2007, cuando el gobierno del Distrito Federal lo despenalizó durante el primer trimestre de embarazo. La despenalización se sustentó en tres argumentos sustantivos: la necesidad de respetar los derechos reproductivos de las mujeres para lograr la ciudadanía femenina plena; la inserción del aborto en el campo de la salud pública, por sus implicaciones en la

8 Traducción propia. 
morbimortalidad y el bienestar integral de las mujeres; la mediación de la justicia social, que obliga a ofrecer dicho servicio médico gratuitamente a las mujeres de menores ingresos, que en el contexto de la ilegalidad carecen del acceso igualitario a un seguro y sanitario procedimiento médico para finalizar el embarazo. El discurso de los derechos reproductivos, es decir, el derecho de las mujeres a la relativa autonomía reproductiva, con seguridad médica y justicia social, evolucionó junto a la conciencia feminista de la segunda ola del último tercio del siglo $\mathrm{xx}$, conciencia que se germinó en las propias contradicciones del modelo industrial tardío y contribuyó a su pronto agotamiento institucional. La aprobación de la reforma de ley en noviembre del 2007 abrió la posibilidad para las feministas y sus aliados de considerar reformas similares en otras partes del país.

Previniendo esto, la constelación antiaborto 9 —aglutinada por la Iglesia católica—adoptó una estrategia a nivel nacional de promover leyes locales de reforma constitucional en los estados, reconociendo el derecho a la vida desde la concepción e incorporando así un término que encierra una visión religiosa de la reproducción a los códigos legales. Dichas leyes se enraízan en discursos eclesiales doctrinarios que comprenden al producto en gestación como una persona falta de protección legal absoluta a lo largo de su evolución prenatal, por ser la expresión más débil de la divina intención. Para principios del 2011, a solo tres años de la despenalización en el

9 La constelación antiaborto está integrada por la Iglesia católica y los agrupamientos civiles asociados; institutos religiosos minoritarios - pero en pleno crecimiento- de índole protestante renacida, como las iglesias evangélicas y pentecostales; el Partido de Acción Nacional (PAN) y sus funcionarios y representantes electos federales y estatales; sectores del Partido Revolucionario Institucional (PRI); representantes individuales del Partido de la Revolución Democrática (PRD) en ciertos estados; y algunos medios de comunicación, particularmente a nivel local.
Distrito Federal, dicha estrategia les abonó resultados positivos en dieciséis estados, con dos más que se sumaron en el 2014 y un precursor —Chihuahua- que se adelantó en 1996.

El debate sobre la propuesta de reforma de ley en el D.F. duró casi medio año, con defensores y opositores informando a las y los congresistas, disputando el significado del aborto en los partidos políticos, los agrupamientos ciudadanos locales, el Congreso de la Nación y los medios de comunicación, de manera que los discursos encontrados sobre el tema permearon la cultura pública de la ciudad capital y el país. Dado que históricamente el aborto nunca ha sido despenalizado en México, sino solo admitido en situaciones excepcionales como la violación, el incesto o el peligro de muerte materna, el cúmulo de nuevas reformas operadas como una cascada contagiosa de desaprobación, ratifica simbólica y jurídicamente el rechazo de dichos gobiernos estatales a la futura posibilidad de despenalizarlo en sus jurisdicciones.

\section{Discursos en defensa de los derechos re- productivos}

En su análisis de los criterios normativos sobre el derecho a la vida en los convenios internacionales de derechos humanos, las determinaciones de sus respectivas comisiones de vigilancia y las decisiones de los órganos jurídicos internacionales de derechos humanos, el Center for Reproductive Rights (Centro para los Derechos Reproductivos [CRR $])^{10}$ de Nueva York identifica el derecho a la vida como un derecho humano fundamental, del cual depende el disfrute de los demás derechos humanos. Asimismo, en su

10 El Center for Reproductive Rights es uno de los mayores socios jurídicos para demandas y negociaciones internacionales del Grupo de Información en Reproducción Elegida (GIRE), la ONG mexicana más antigua y especializada en el tema. 
publicación intitulada ¿El derecho a la vida de quién? ${ }^{11}$ (18), se hace hincapié en que la jurisprudencia internacional en materia de derechos humanos reconoce dicho derecho únicamente a partir del nacimiento: "La ley internacional de derechos humanos de las mujeres, los cuerpos internacionales y regionales de derechos humanos y altas cortes nacionales han establecido que las protecciones prenatales deben compaginarse con los derechos humanos de las mujeres" (p. 2).

Por lo mismo, la actual tendencia religiosa conservadora mexicana de extender las garantías jurídicas del derecho a la vida al pleno periodo prenatal y, especialmente, "proteger la vida desde la concepción", se interpreta por el discurso del CRR como una estrategia calculada, que a nombre de la tutela absoluta del producto en gestación niega a las mujeres el rango amplio de derechos y servicios reproductivos a que tienen derecho y de que dependen su derecho a la vida, la salud, la dignidad, igualdad, autonomía y libertad, entre otros. El CRR sostiene: "Cuando los estados protejan el derecho a la vida antes de nacer sin considerar los derechos de las mujeres, se arriesgan los derechos fundamentales de las mujeres, mientras que se hace poco para proteger la vida prenatal" (18, p. 3). En este sentido, las leyes de derechos a la vida desde la fecundación son señaladas por este discurso como el primer paso hacia la meta de progresivamente minar el propio concepto de los derechos reproductivos y los acuerdos internacionales y nacionales que los reconozcan.

No hay consenso en torno a cuándo se inicia la vida humana. Ni los convenios internacionales de derechos humanos, ni las leyes de protección absoluta al cigoto-embrión-feto precisan definiciones de textos sagrados, filosóficas o científicas que lo establezcan.

11 Título original: Whose right to life? Women's rights and prenatal protections under human rights and comparative law (traducción de la autora).
El documento del CRR enfatiza que aun las leyes que protegen el producto desde la fecundación no definen el inicio de la vida humana, sino que solo atestan el momento en que jurídicamente se considera el gestado como una persona, sujeto de derechos. El CRR afirma que especialistas internacionalmente reconocidos en interpretación de los derechos humanos, así como los propios registros de las negociaciones de los tratados de dichos derechos, establecen que el reconocimiento del derecho a la vida en los distintos convenios internacionales no abarca a la vida prenatal. Asimismo, se señala que los comités oficiales de monitoreo del cumplimiento de estos tratados continuamente han enfatizado la importancia de garantizar los derechos de las mujeres, en particular el derecho a la vida y la salud. Por ejemplo, el Comité que vigila el cumplimiento de la Convención por la Eliminación de la Discriminación Contra las Mujeres (CEDAw, por su nombre en inglés), ha expresado en sus resoluciones su preocupación por la influencia negativa sobre dichos derechos de leyes muy restrictivas de prohibición del aborto (19).

En respuesta a dos informes mexicanos anuales, en el 2012 el Comité CEDAW emitió un análisis y recomendaciones en torno a la situación nacional, expresando su preocupación por las nuevas reformas constitucionales locales que en distintos estados reconocen el derecho a la vida desde la fecundación. El Comité también manifestó su consternación por la denegación por parte de proveedores de servicios médicos y trabajadores sociales del acceso al aborto despenalizado para mujeres embarazadas que cumplan los restringidos criterios reglamentarios, y "a continuación, las denunciaron ante las autoridades judiciales, quienes a su vez les condenaron a largas penas de prisión por infanticida asesinato". Como antecedente, el Comité de la CEDAw había determinado en el 2006 que la negación de servicios de 
salud exclusivos para mujeres equivale a discriminación (19).

Por considerar el aborto despenalizado y seguro un factor esencial del derecho a la vida, la salud, la integridad física, la vida privada, la no discriminación y la autonomía reproductiva de las mujeres, el Grupo de Información en Reproducción Elegida (GIRE) recuerda que dichos derechos están consignados en la Constitución Política de los Estados Mexicanos y en tratados internacionales de derechos humanos ratificados por México (20). ${ }^{12}$ El GIRE, destacando el vínculo entre el respeto a los derechos humanos de las mujeres y el acceso gratuito a los servicios del aborto legal, enfatiza su ubicación en el campo de la salud, ${ }^{13}$ destacando que la gratuidad del servicio se inscribe en el rubro de la justicia social. Desde este enfoque, dicho servicio contempla la deuda sociopolítica con las mujeres pobres, menos educadas e indígenas que actualmente "tienen nueve veces más probabilidad de tener un aborto inseguro que mujeres con mayores posibilidades económicas, más educadas y que no pertenece a etnias indígenas” (20, p. 3).

También el discurso de la justicia social involucra a otro gran adeudo de la actualidad en

12 Fundada en 1991, GIRE es una asociación civil mexicana especializada en materia de derechos reproductivos, que de mandato principal se asume "generar, sistematizar y difundir información relativa a los derechos reproductivos para que estos sean reconocidos y respetados en México". Su enfoque en la problemática del aborto en México ha producido investigaciones sobre el tema, a la vez que se proporciona asesoría jurídica en las situaciones y a los casos meritorios.

13 Según datos de la Secretaría de Salud Pública, en el 2010 el aborto penalizado fue la causa del $11 \%$ de las muertes maternas en México. Asimismo, las condiciones inseguras e insalubres de la clandestinidad han significado serios problemas de morbilidad para las mujeres; solo en el 2009 se registraron 159005 admisiones hospitalarias por complicaciones de salud por abortos inseguros (Juárez y Susheela, nota 9, citado en [20]). relación con estos sectores de mujeres, que por ser socialmente más vulnerables experimentan mayor frecuencia de criminalización, con penalidades más largas por abortar. Esto se constata con las fuertes sanciones jurídicas recibidas por mujeres pobres e indígenas en muchos estados por haber abortado o por ser sospechosas de haber practicado un aborto. ${ }^{14}$ $\mathrm{Y}$ esto es a pesar de que en muchos casos las mujeres procesadas insisten en que fueron abortos espontáneos. Aun cuando el aborto es penalizado en todo el país, con excepción de casos de violación sexual o en el D.F., las nuevas leyes del derecho a la vida desde la concepción en diecinueve estados de la República han intensificado la persecución de mujeres que recurran a los servicios públicos de salud para atender efectos físicos negativos de un aborto intencional —o espontáneo-en condiciones ilegales, inseguras e insalubres. Este es el caso de las 679 mujeres que han sido denunciadas por empleados hospitalarios y profesionales médicos por haber abortado entre el 2009 y el 2011, lo que suma a 226,3 mujeres por año (20).

El discurso a favor del sentido amplio de los derechos reproductivos menciona concretamente dos leyes mexicanas que, según su interpretación, garantizan el acceso al aborto despenalizado. La NOM 046 (violencia familiar, sexual y contra las mujeres), publicada en abril del 2006, obliga a las instituciones locales del Sistema Nacional de Salud a prestar dicho servicio a mujeres violadas. Producto de la solución amistosa entre la Corte Interamericana de Derechos Humanos y México en el 2007, en relación con la demanda interpuesta en el 2000 por el GIRE y el Center For Reproductive Rights por la denegación oficial del aborto legal a una niña indígena inmigrada a Baja Califor-

14 Según la investigación de GIRE, durante el 2007 y el 2012 un total de 171 personas fueron consignadas por el aborto en 19 estados de la República, con 151 procesos penales y 127 consignaciones (20). 
nia y violada en su casa por un drogadicto, la NOM 046 tiene como propósito remediar las arbitrariedades profesionales y estatales con que el derecho al aborto por violación fue ignorado en este caso. De tal manera, la solución amistosa encierra una serie de acuerdos que facilitaron la reformulación de la ley y la capacitación de servidores públicos en materia de los derechos reproductivos de las mujeres.

Por su parte, la Reforma Constitucional en Materia de Derechos Humanos, del 2011, establece que toda persona gozará de los derechos humanos garantizados en la Constitución y en los tratados internacionales suscritos por México, haciendo explícito que la interpretación de dichos derechos en la Constitución o en los tratados debe favorecer a las personas con "la protección más amplia”. De esta manera, se considera a ambos - la Constitución y los tratados internacionales - al mismo nivel jerárquico en el marco de la jurisprudencia constitucional nacional, siendo el de mayor valor el que en un caso concreto brinde a las personas la protección más amplia. A su vez, "queda prohibida toda discriminación motivada por origen étnico o nacional, el género, la edad, las discapacidades, la condición social, las condiciones de salud, la religión, las opiniones, las preferencias sexuales, el estado civil o cualquier otra que atente contra la dignidad humana y tenga por objeto anular o menoscabar los derechos y libertades de las personas" (Constitución Política de los Estados Unidos Mexicanos [21]). En cuanto a la discriminación de género, el discurso de defensa de los derechos reproductivos plantea que la falta de plena autonomía reproductiva ejemplifica la discriminación contra las mujeres.

\section{Discursos a favor de la personificación prenatal desde la concepción}

En México, la Iglesia católica y las asociaciones civiles cercanas encabezan el archipiélago de actores conservador-tradicionalistas que se oponen a la despenalización del aborto. Con el 83,9\% de la población nacional identificándose como católica (22), ${ }^{15}$ los discursos eclesiales sobre el tema y otros asociados - como el derecho a la vida, el género, el cuerpo, la sexualidad, la reproducción y la familia- construyen la mirada contrapuesta al discurso de los derechos reproductivos. A pesar de ser un tema de interés para la Iglesia desde épocas tempranas, distintos discursos han informado sus posiciones sobre el aborto durante siglos. Sin duda, los reclamos feministas desde la década de los setenta, que vincularon la ciudadanía femenina sustantiva con la autonomía corporal, así como la institucionalización en los años noventa de los derechos reproductivos y sexuales como componentes indivisibles del paradigma de los derechos humanos, ${ }^{16}$ fomentaron la producción y afinación de discursos contrarios y la consiguiente movilización militante de actores eclesiales institucionales y cívicos a favor de una visión societal profundamente distinta.

Desde dicha perspectiva de sustancia patriarcal, ${ }^{17} \mathrm{el}$ aborto se inserta en la tensión religiosa binaria entre el espíritu y la carne, con el valor del espíritu edificado sobre las tentaciones carnales, las que son comprendidas como pruebas, faltas, limitaciones o

15 Según el Instituto Nacional de Estadística y Geografía (Inegi), en México se suman a 92,9 millones de católicos de cinco años y más.

16 La Conferencia Mundial de Derechos Humanos, celebrada en Viena en 1993, reconoció a todos los derechos específicos de las mujeres como derechos humanos indivisibles y no jerárquicos, tal y como los demás derechos humanos.

17 Hace décadas que Rubin (24) criticó el concepto de patriarcado por su generalidad ahistórica. Lo uso aquí en su sentido más llano, refiriéndome a un asimétrico régimen de género de estatus y privilegios masculinos, profundamente institucionalizado e individualmente incorporado en lo hondo del inconsciente, los gestos corporales, todas las relaciones sociales y las acciones cotidianas. 
perversiones humanas. Inmersas en la tensión entre la esencia espiritual y las pujantes pulsaciones del cuerpo, las mujeres simbolizan el peligro del dominio de los placeres carnales. Por medio de Eva, la figura bíblica del mito de origen judeocristiano asumido por el catolicismo, la mujer es representada como naturaleza pura, potencialmente indómita, siempre sospechosa de desbordar las fronteras sociales que sujeten los apetitos instintivos, falta de disciplinamiento, vigilancia y dominación (23). Con referencia a esto, Bourdieu recuerda que en las culturas androcéntricas el pundonor masculino depende del comportamiento sexual de la mujer, lo que por un lado deviene en los controles normativos, físicos y simbólicos orientados al género femenino, pero por el otro, ilumina un capital social simbólico con el que las mujeres navegan las aguas de la dominación masculina (12). En la mitología católica, el pecado original simboliza el tránsito del estado espiritual al mundo material, con mayor y menor responsabilidad según género por la caída de gracia y propuestas corporeizadas — también según género- de la reconquista espiritual.

Según la interpretación del Génesis en la Teología del cuerpo, una serie de catequesis emitidas por Juan Pablo II entre 1979 y 1984 en sus audiencias de los miércoles, publicadas en el 2005, la vergüenza del pecado original se subsana mediante la creación consagrada en la comunión corporal entre hombre y mujer, que en el matrimonio devela en el cuerpo femenino el misterio sagrado a partir del orden natural de complementariedad de los sexos (25). La creación humana que deviene del encuentro sexual entre el hombre y la mujer en el matrimonio re-espiritualiza lo corpóreo, iluminando la intención divina subyacente al hecho creativo. La complementariedad de los rasgos identitarios sexuales no se comprende - como afirma el discurso feminista - como una construcción cultural, sino que "forma parte constitutiva de la persona y la define de manera esencial" (25, p. 103).

Desde esta mirada, la maternidad simboliza el ordenamiento divino, amparado por la ley natural, que descansa en atributos y labores según sexo, también naturales, las cuales informan un orden de género y arreglos familiares que son considerados esencialmente ahistóricos y presociales. La categoría familia natural engloba a dichas identidades relacionales en el matrimonio, siendo la procreación el "primer fin o propósito" de un orden familiar "grabado en nuestra naturaleza", que actualmente "permanece reprochada y bajo amenaza en el siglo 21" (26). La perspectiva de la familia es el vehículo conceptual que compite con la perspectiva de género, edificando a la familia natural como el núcleo básico de la sociedad, anterior al Estado: "un sistema familiar ideal, óptimo y verdadero" (26) que comprende a los individuos a partir de su pertinencia y funciones familiares, sustentado en "un orden moral objetivo que excluye el recurso de la contracepción, la esterilización y el aborto" (27, p. 3).

La posición de la Iglesia católica de rechazo absoluto al aborto se ha preservado desde 1869, cuando el papa Pío IX publicó el acta Apostólica Sedis que lo define como un homicidio en todas las circunstancias a lo largo del embarazo y lo clasifica como pecado grave y razón de excomunión. La premisa de la hominización inmediata - es decir, la entrada del alma al cuerpo al momento de la fecundación- informa a dicha posición. Empero, en una investigación sobre la historia del aborto en el catolicismo y los discursos respectivos de la jerarquía católica mexicana, Católicas por el Derecho a Decidir (CDD) describe cómo otras premisas de hominización retardada han sustentado históricamente interpretaciones que no lo penalicen sino hasta un tiempo después del inicio del embarazo. Santo Tomás de Aquino 
(1127-1174), por ejemplo, mantuvo que en razón de la hominización tardía no se consideraba la interrupción del embarazo como un aborto durante los primeros cuarenta días para embriones masculinos y los ochenta días para embriones femeninos (28). Por su parte, san Agustín (354-430) afirmó que hasta tiempo después de la fecundación "el acto del aborto no se considera homicidio porque aún no se puede decir que hay un alma viva en el cuerpo que carece de sensación" (29, p. 7). Con la creciente secularización de la era moderna, la tendencia de asociar el aborto con el pecado de la sexualidad fuera del matrimonio se sustituyó por el dilema de la corporalidad del alma y la entonces certeza de su realización al momento de la concepción. Esta última preocupación recuerda de nuevo la tensión en el campo católico entre el espíritu y la materia, la cual desde el siglo XIX se resuelve mediante el recurso de la hominización inmediata de la formación celular primaria.

A partir de dicha posición y en el marco de la Cuarta Conferencia de la Mujer en Beijing en 1995, el papa Juan Pablo II emitió su encíclica Humanae Vitae, en la cual reconfirma la obligación religiosa de las y los creyentes de conceder al acto conyugal íntimo el propósito procreador (29), desaprobando la anticoncepción y los condones y condenando, en términos absolutos, cualquier forma o causa de aborto como "un desorden moral grave, en cuanto eliminación deliberada de un ser humano inocente" (30). Dicha representación personificada del embrión como un ser humano inocente también se acompaña visualmente de manera emblemática en la propaganda antiaborto contemporánea, en donde la imagen del feto figura como un ente individuado de la madre, una persona aparte, el sujeto verdadero del embarazo (31). Recalcando dicha noción, el arzobispo Norberto Rivera y ocho obispos auxiliares de la Arquidiócesis de México expidieron la siguiente declaración en el 2008, con respecto a la ratificación de parte de la Suprema Corte de Justicia de la Nación (SCJN) de la reforma de salud del D.F. que despenalizó el aborto durante las primeras doce semanas de embarazo: "No se puede justificar el aborto pretendiendo negar el estatuto humano del embrión. El ser humano debe ser respetado y tratado como persona desde el instante de su concepción [...]" (28, p. 194).

Desde la década de los noventa, la estrategia del actor conservador religioso se ha globalizado, nutriendo redes interreligiosas, nacionales e internacionales. Su dinamismo está a la vista: organizando seis Congresos Mundiales de la Familia que afirman e impulsan la familia natural, ${ }^{18}$ elaborando la perspectiva de la familia; impulsando a nivel nacional e internacional el Día de la Familia y el Día

18 La Primera Conferencia Mundial de la Familia (CMF), llevada a cabo en Praga en 1997, registró más de 700 delegados de 200 organizaciones de 43 países, y centró sus esfuerzos en la reconstrucción de la familia tradicional en los países postcomunistas. La Segunda Conferencia, realizada en Ginebra en 1999, congregó a 1600 delegados de 270 organizaciones de 65 naciones, quienes analizaron y elaboraron estrategias globales con respecto a lo que consideran la política antifamilia de los diferentes organismos de la ONU. La tercera CMF, llevada a cabo en México D.F. en el 2004, reunió a 3300 delegados de 75 países, con el pleno aval y participación del entonces gobierno conservador del Partido de Acción Nacional (PAN); en aquella oportunidad se avanzó en la elaboración de la perspectiva de la familia y la estrategia antiaborto, fijando el domingo inmediatamente antes del Día Internacional de la Mujer (el 8 de marzo), como el Día de la Familia. La Cuarta Conferencia, que se hizo esta vez en Varsovia en el 2007, con más de 3900 delegados, enfocó sus trabajos en las consecuencias del invierno demográfico, precisando estrategias nacionales y globales para la repoblación del mundo. La Quinta Conferencia, que tuvo lugar en Ámsterdam en el 2009, elaboró estrategias nacionales e internacionales de institucionalización de la familia natural. La última conferencia, celebrada en Madrid en el 2012, examinó los peligros del feminismo, la perspectiva de género y el matrimonio entre personas del mismo sexo, y recomendó que la salida de las mujeres del trabajo remunerado y su retorno al hogar sea la respuesta al desorden familiar contemporáneo. 
del No Nacido; ${ }^{19}$ promoviendo leyes a nivel estatal y nacional en países como México, para que en gran parte del territorio nacional reconozcan la personificación del embrión desde la fecundación; criminalizando el aborto en casi todas las circunstancias; ilegalizando métodos de anticoncepción masivamente empleados junto a la medicina celular con embriones; finalmente, disputando la perspectiva de género y la institucionalización de los derechos reproductivos y sexuales en los institutos inter y supra estatales, como la ONU.

Este es el sentido de la reflexión del obispo de Querétaro, Mario De Gasperín Gasperín, que se opone abiertamente a dicha perspectiva: "Con el pretexto de luchar contra el machismo y la discriminación de la mujer (que buena falta nos hace), negando el hecho biológico y privilegiando el cultural, se reinventa la noción de género (ideología de género, equidad de género, etcétera) la cual hace referencia no solo a los dos sexos, sino (a) toda una constelación: masculino, femenino, homosexual-lesbiano, bisexual, transexual, etcétera, dando carta de ciudadanía a la promiscuidad y a la degradación sexual [...]" (28, p. 186).

Así es que, de manera lúcida y precisa, el obispo apunta a algunas de las diferencias principales entre las dos posturas que hoy en día contienden por los significados culturales en torno al aborto, destacando el desencuentro entre interpretaciones biologistas y culturalistas de las identidades sexuales y la relación jerárquica entre ellas, es decir, entre el sexo y el género. Divergencia que asimismo remite a la distancia entre la familia natural y lo que Beck (10) llama la familia postfamilia, para referirse a las múltiples variantes de composición, membresía y división de trabajo de la unidad familiar

19 Un ejemplo reciente es el de Nuevo León, cuyo Congreso estatal (en marzo del 2015) designó el 25 de marzo de cada año como el Día del Niño por Nacer. contemporánea. Esto conlleva miradas societarias que encierran distintas propuestas de moralidad, valores y jurisprudencia, contrastados modelos de la familia, imaginarios sociales - de mayor o menor uniformidad o pluralidad-y nociones de la convivencia colectiva. Lo que finalmente se reduce a una diferencia fundamental en cómo se concibe el Estado, su función y los márgenes de su relación con la religión.

\section{Recapitulación y perspectiva}

La disputa sobre los significados del aborto no es particular de México, sino que invade el campo político contemporáneo de muchos países de distintas culturas, historias y perfiles religiosos; asimismo, agita las discusiones, evaluaciones y renovaciones de convenios en institutos interestatales como la ONU. Discursos representativos de las posiciones enfrentadas construyen distintas nociones de la vida, el momento - y la autoridad- que define la personificación, la noción y los sujetos de la ciudadanía, los derechos humanos, los valores que orientan las instituciones e informan el código legal, la moralidad, la sexualidad, el matrimonio o el emparejamiento y su relación con la reproducción biológica, las identidades de género, sus roles y la organización de la familia, y finalmente, el perfil del Estado y su relación con la religión. Como constituyentes de la formación discursiva de esta última modernidad, las posiciones encontradas sobre el aborto son emblemáticas de una contienda mayor, propia de la presente etapa histórica de transición entre dos fases capitalistas, dos modelos estructurales-tecnológicos distintos, la modernidad industrial y la era postindustrial globalizada. A su vez, dicha contienda por la interpretación cultural del actual contexto histórico de desfase entre contenidos y formas institucionales rebasadas y cotidianidades movedizas y cambiantes, 
contrapone discursos, actores colectivos, estrategias y agencias que posicionan el cuerpo reproductor de las mujeres - y el cuerpo de deseo- como territorios centrales en la definición del rumbo del proyecto societario.

¿Cuáles son las implicaciones de esto para México? La reciente disputa sobre la despenalización del aborto en los estados de Guerrero y Nuevo León en el 2014, ha elevado a diecinueve los estados, de 32 en total, que reconocen el derecho a la vida —es decir, la personificación-desde el momento de concepción, con seis estados más en distintas etapas de reformas constitucionales similares. Por otro lado, la despenalización del aborto durante el primer trimestre en el D.F. cumplió su octavo año en marzo del 2015, con 138792 intervenciones seguras y sanitarias. Dos discursos contrastados que resultan en dos modalidades de biopolítica, ${ }^{20}$ dividiendo el mapa nacional en franjas de marcos jurídicos y prácticas médicas diferenciadas en torno a los derechos reproductivos y los derechos humanos de las mujeres. Mientras que los actores del discurso antiaborto consideran exitosa su estrategia y formulan la meta de revertir la despenalización para evitar lo que consideran un genocidio, los actores opuestos a los avances

20 Foucault define a la biopolítica como unos de las técnicas centrales de la dominación de la modernidad. A diferencia de la microfísica, cuyos disciplinamientos minuciosos e incesantes del cuerpo individual producen cuerpos dóciles y útiles, la biopolítica es una técnica de poder enfocada en el cuerpo-especie y asociada al concepto de población que emergió a finales del siglo XVIII. La biopolítica se ocupa de los procesos socio-biológicos que marcan la vida humana colectiva, la sexualidad - aceptada y sancionada-, los embarazos - llevados a término o interrumpidos-, los nacimientos — considerados legítimos e ilegítimos-, la tasa de natalidad, la edad de los matrimonios, las prácticas anticonceptivas y la muerte, entre otros. En fin, fundamental a la constitución de una economía política de población, dicho dispositivo de poder moldea la demografía del cuerpo-especie mediante "una serie de intervenciones y controles reguladores: una biopolítica de la población" (1, pp. 25-36). de las políticas de derecho a la vida desde la concepción censuran el incumplimiento de los convenios internacionales suscritos por México sobre los derechos humanos de las mujeres y, asimismo, la inobservancia de la Reforma Constitucional mexicana en Materia de Derechos Humanos del 2011, que claramente fija la jerarquización jurídica de leyes nacionales o convenios internacionales suscritos por México, según lo que mejor ampare los derechos de los individuos, en este caso, de las mujeres. Y en medio de la tensión entre un discurso y otro, lo que resalta de manera innegable es la creciente influencia religiosa en el quehacer legislativo y jurídico de la Nación, lo que sugiere la posibilidad de hablar de una fase post-laica en cuanto al carácter del Estado mexicano, en claro contraste con el perfil del Estado mexicano postrevolucionario.

\section{Referencias bibliográficas}

1. Foucault M. Historia de la sexualidad I. La voluntad de saber. México: Siglo XXI; 1977.

2. Maier E. La disputa por el cuerpo de la mujer, la/s sexualidad/es y la/s familia/s en Estados Unidos y México. Frontera Norte. 2008 julio-diciembre; 20.

3. Maier E. El aborto: eje de la disputa por los significados culturales. La Alijaba, segunda época. Revista de Estudios de la Mujer. 2010; 14. Argentina: Universidad de La Pampa, Comahue y Luján.

4. Maier E. Documenting Mexico's Culture War. Latin American Perspectives [internet]. 2012; 39 (6): 155-164. Disponible en: « http://lap.sagepub. com/content/early/2012/08/29/0094582X12456680. abstract?rss=1».

5. Maier E. Guerra cultural USA. Significando la persona, la familia y el Estado entre la fe y la ciencia. En Olmos M, coordinador. Fronteras culturales, alteridad y conflicto. Tijuana, México: El Colef; 2013. pp. 190-227.

6. Maier E. Disputando los significados culturales en el ocaso de la modernidad industrial: cuerpos, personas, derechos y ciudadanías en el México contemporáneo. En Tarrés ML, Maier E, López S, et al., coordinadores. 15 años de políticas de igualdad: Los avances, los dilemas y los retos. México: El Colef/Colmex; 2014.

7. Castells M. Communication Power. Oxford, New York: Oxford University Press; 2009.

8. Bauman Z. Vida líquida. Barcelona: Paidós Ibérica; 2005. 
9. Touraine A. ¿Podremos vivir juntos? La discusión pendiente: El destino del hombre en la aldea global. Buenos Aires: Fondo de Cultura Económica; 1997.

10. Beck U. La democracia y sus enemigos. Barcelona: Paidós; 2000.

11. Hunter J, Wolfe A. Is There a Culture War? A Dialogue on Values and American Public Life. Florida, USA: Pew Forum and the Brookings Institution; 2006.

12. Bourdieu P. La dominación masculina. Guadalajara: Universidad de Guadalajara; 1996.

13. Portantiero JC. Los usos de Gramsci. México: Folios Ediciones; 1981.

14. Hunter JD. Culture Wars: The struggle to define America. Nueva York: Basic Books; 1990.

15. Castells M. El poder de la identidad, volumen II. México: Siglo XXI; 1999.

16. Gutiérrez S. Discurso político y argumentación Ronald Regan y la ayuda a los "contras". 2005. Ticom 50, Cuadernos del Taller de Investigación en Comunicación masiva. Universidad Autónoma Metropolitana.

17. Alvarez S. Translating the Global: Effects of Transnational Organizing on Local Feminist Discourses and Practices in Latin America. Meridians. 2000; 1 (1): 29-67. Northampton: Indiana University Press.

18. Centre for Reproductive Rights. Whose right to life? Women's rights and prenatal protections under human rights and comparative law [internet]; 2013. Disponible en: «http://reproductiverights.org/en/ document/whose-right-to-life-womens-rights-andprenatal-protections-under-human-rights-andcomparative-law» [consultado: 15-08-2014].

19. Comité para la Eliminación de la Discriminación contra la Mujer (CEDAW). Observaciones finales del Comité para la Eliminación de la Discriminación contra la Mujer. Chile, 36으 periodo de sesiones; 2006, párrafo 19 [CEDAw/C/CHI/CO/4] [internet] [consulta: 21 de octubre del 2014]. Disponible en: http// bit.ly/moh2hx.
20. Grupo de Información en Reproducción Elegida (GIRE). informe.aborto._pdf.pdf-Adobe Reader; 2013.

21. Constitución Política de los Estado Mexicanos; 2001.

22. Instituto Nacional de Estadística y Geografía (Inegi). 2010.

23. De Beauvior S. El segundo sexo. Los hechos y los mitos, volumen 1. Buenos Aires: Siglo XX; 1981.

24. Lamas M. El género: la construcción cultural de la diferencia sexual. Editorial Porrúa. Universidad Nacional Autónoma de México. 1996.

25. Rodríguez G. Frenar la concupiscencia: la educación sexual en el discurso eclesial. En Aldaz E, Mejía M, coordinadoras. De la brecha al abismo. México: Católicas por el Derecho a Decidir; 2013. pp. 95-124.

26. Primer Congreso de la Familia. La Familia Natural: Un manifiesto; 2004.

27. Carta de los Derechos de la Familia. Vaticano, Roma; 1987.

28. Aldaz E, Mejía M. El vientre de la mujer es el lugar más inseguro de la Tierra: El discurso de los obispos sobre las mujeres y el aborto. En Aldaz E, Mejía M, coordinadoras. De la brecha al abismo. México: Católicas por el Derecho a Decidir; 2013. pp. 175-200.

29. Hurst J. La historia de las ideas sobre el aborto en la iglesia católica. Lo que no fue contado, quinta edición. México: Católicos por el Derecho a Decidir; 1998.

30. Juan Pablo II. Evangelium Vitae; 1995.

31. Amucháustegui A. Las mujeres en el discurso de los obispos. En Aldaz E, Mejía M, coordinadoras. De La brecha al abismo. México: Católicas por el Derecho a Decidir; 2013. pp. 125-134. 